\title{
Cell Phones and their Impact on Male Fertility: Fact or Fiction
}

\author{
Alaa J. Hamada, Aspinder Singh and Ashok Agarwal* \\ Center for Reproductive Medicine, Cleveland Clinic, Cleveland, Ohio, USA
}

\begin{abstract}
Today, cell phone technology is an integral part of everyday life and its use is not only restricted to voice conversations but also conveying news, high resolution pictures and internet. However, these advances in technology are accompanied by progressive boost in the intensity and frequency of the emitted electromagnetic waves without consideration of their health consequences. Our bodies act as parasitic antennas that receive these waves and convert them into electric and magnetic fields. While thermal effects at the present level of cell phone radiation are negligible, most of the biological interactions are attributed to non-thermal effects. Male reproductive system is highly compartmentalized and sensitive biological system that requires the integration of intrinsic and extrinsic factors to properly function. The generated electrical currents may alter the hormonal milieu and testicular microenvironment, necessary for sperm production. Additionally, sperm are electrically active cells and their exposure to cell phone electromagnetic waves and currents may affect their motility, morphology and even their count. Leaky plasma membranes, calcium depletion and oxidative stress are the postulated cellular mechanisms mediating the harmful effects of cell phones radiation on sperm and male fertility potentials. Evidences for such impacts come from designed animal and in vitro studies which may be different from in vivo human exposure. Nevertheless, the important advice is to apply strict regulations on further increase in the power density of the emitted cell phone radiation and to conduct in vivo human research to study its negative effects on fertility.
\end{abstract}

Keywords: Cell phone, electromagnetic waves, male infertility, sperm, calcium, reactive oxygen species, plasma membrane.

\section{INTRODUCTION}

In March of 1876 Alexander Graham Bell was awarded the first patent for an electronic telephone [1]. Since that invention, the telephone has seen many technological advances. Touchtone phones, wireless hand-sets, car-phones, and most recently the cell phone and smartphones have all emerged in response to societies' needs and desires. Today, cell phone technology is an integral part of everyday life, and its use will continue to grow as providers proceed to offer more expansive services and newer, better products.

However, the implications of this technology on human health are often overlooked. Innovations in cell phones may be associated with detrimental effects on the human brain, cardiovascular system, and more specifically male reproduction. This article will analyze the means of cell phone interference on normal bodily functions and will take an in depth look at their relations with male reproductive health.

We will begin by analyzing the biophysics of cell phones, which will then be followed by an examination of the cell phone radiation effects on biological tissues. Then specific effects on semen and male fertility will be scrutinized, and we will round out with a discussion of the complications and ambiguities surrounding cell phone research.

*Address correspondence to this author at the Lerner College of Medicine, Andrology Laboratory, Cleveland Clinic, Desk A19.1, 9500 Euclid Avenue, Cleveland, Ohio 44195, USA; Tel: (216) 444-9485; Fax: (216) 445-6049; E-mail: agarwaa@ccf.org

\section{BIOPHYSICAL PROPERTIES OF CELLULAR PHONE ELECTROMAGNETIC RADIATION}

Cellular phones are defined as devices emitting radiofrequency electromagnetic waves (RF-EMW). These waves transmit signals from the cellular phone to the base stations and antennas. The frequency of such waves is low and ranges from 800-2200 MHZ. However there is still risk to the human user, because our bodies can act as antennas that absorb these waves and convert them into eddy currents [2, 3].

The mechanisms of cell phones operate in such away that the sound wave produced from the speaker goes through a transmitter that converts the sound into a sine wave. This sine wave then travels to the antenna, which then projects the wave out into space. Average power usage of the transmitter is about 0.75 to 1 watt, with a maximum of $2 \mathrm{~W}$. The propulsion of the electric sine wave running through the transmitter circuit also yields an electromagnetic field. As the electric current oscillates back and forth, these electro-magnetic fields continue to build up and collapse, resulting in electromagnetic radiation [3].

The preliminary cell phone system, Analogue NMT (Nordic Mobile Telephone) system, was introduced in the 1980 s, and operated at an electromagnetic resonance of 902.5 MHz. A decade later, the GSM (global system of mobile communications) succeeded it, operating at a radiofrequency of $902.4 \mathrm{MHz}$, pulsing at $217 \mathrm{~Hz}$. The most recent DCS (digital cellular system) operates at a radiofrequency of $1800 \mathrm{MHz}$ [4]. Advances in cell phone telecommunication systems are obviously associated with an increasing signal 
frequency, which correlates with higher energy radiofrequency waves.

Networks used by specific countries differ in the transmission frequencies for the radio waves. European and Asian countries operate at $850 / 900 \mathrm{MHz}$, whereas in United States mainly networks operate at 1800/1900 MHz. Increased frequency means increased energy in the waves. Furthermore, increasing globalization has yielded cellular phones which can function in multiple countries, and over all four frequencies $(850 / 900 / 1800 / 1900 \mathrm{MHz})$, thereby appropriately termed quad-band phones.

In order to measure the impact of radio frequency electromagnetic waves on the human body a standardized unit called the SAR value (Specific Absorption Rate) was established. The SAR measures the rate of radiofrequency energy absorption in the body, expressed as watt $/ \mathrm{Kg}$. Device specific SAR tests are conducted at the highest power level of the device, in all four frequency bands. Since 1996, the FCC (Federal Communication Commission), has limited the maximum legal SAR of any handheld mobile device to 1.6 watts per kilogram, and from the year 2000 onwards, all cell phone manufacturers must place labels on their phones providing their radiation levels [5]. Each country has its own regulatory agency which establishes health parameters for RF-EMW emitting devices.

Lastly, the actual SAR value arising from a wireless device may differ from the reported maximum. This is due to multiple factors such as proximity of the cell phone to the body, usage mode (talk versus standby mode), and the use of hands-free (Bluetooth) devices [5].

\section{INTERACTION OF CELL PHONE RADIATION WITH BIOLOGICAL TISSUES}

\section{Cell Phones Have a Dual Effect on the Human Body}

Electromagnetic waves (EMW) emitted from cell phones and even microwaves oven fall within the low frequency range of EMW between $300 \mathrm{MHz}$ to several gigahertz. Such level is far below the high frequency EMW of X-ray and gamma rays. EMW travel through space at the speed of light, however, their energy level depends on their frequency and wavelength. The energy carried in EMW is composed of electrical and magnetic fields and it is better represented by the term power density (PD). PD is defined as the amount of power per unit area in a radiated microwave field and is usually expressed in milli- or microwatts per square centimeter $\left(\mathrm{mW} / \mathrm{cm}^{2}\right.$ or $\left.\mu \mathrm{W} / \mathrm{cm}^{2}\right)$. Nevertheless, the level of energy in such EMW is so low that it cannot break the covalent bonds in biological molecules [6]. This type of radiation effect on molecular level is called non-ionizing radiation to differentiate it from the ionizing radiation effect of high frequency EMW.

In general, the exposure to EMW from different sources is divided into two categories: "continuous" and "pulsed" according to the characteristics of the emitted waves. The biological effects of pulsed wave exposure are even more harmful than that of continuous variety from other sources.

As a rule, the coupling of electrical field and magnetic field of EMW outside the biological system will no longer be sustained inside the body due to the electrical and magnetic properties of living tissues [7].

The human body acts as parasitic antenna that receives the EMW from external sources [6]. Specifically, both electrical fields and magnetic fields can induce electrical fields and currents inside living tissues. However, the generated internal electrical currents are of much lower strength and of different directions from the external ones. These alterations in strength and directions reflect the electrical properties of human body, such as the permittivity and conductivity. To understand these properties, human tissue is best described as lossy medium with dielectric properties due to high content of water in addition to other organic molecules and ions. When living tissue is exposed to EMW the dielectric (dipole) molecules will be polarized, the extent of such polarization is called permittivity [8]. Conductivity, on the other hand, describes the conduction current density produced by an applied electrical field [6].

Essentially, the high water content renders human body poor conductor to the applied electrical field. In contrast, the applied magnetic field is easily transmitted through human body and this property is called permeability [6]. Moreover, magnetic field act as another source of induced alternating currents inside the human body. Thermal and non-thermal effects are the main mediators of EMW interaction with biological system and both the electrical and magnetic properties determine sequelae of such effects on human body. We will explain these effects with a particular emphasis on male reproductive cells.

\section{Thermal Effects}

The tissue temperature increase resulting from exposure to EMW is referred to as "thermal effects". Heat is primarily associated with absorption of high frequency EMW radiation resulting from enhanced electrical conductivity of the tissue media. Thermal effects may cause disruption of cell function and development [9]. Increment of tissue temperature in an organ is related to imbalance between heat generation and heat dissipation. Heat generation depends on SAR and energy level (power density) of emitted EMW which must exceed $100 \mathrm{~mW} / \mathrm{cm}^{2}$ to have heating impact on biological tissues [6].

In contrast, heat dissipation involves three mechanisms: heat conduction to other tissues, convection through blood perfusion, and radiation to the surroundings. Generally, the two most vulnerable organs to thermal effects are the eyes and testes because of limited capacity of heat dissipation. However, the power density of the cell phone EMW and SAR is so low that the increment of body tissue temperature is negligible at the current level of frequency and energy of EMW in modern cell phones [6].

\section{Non-Thermal Effects}

The vast majority of the sustained cell phone EMW related biological consequences can be explained by "Nonthermal effects". These effects include all the interactions of EMW with biological tissues without production of heat or a measurable rise in temperature. Specifically, the magnetic field, rather than the electrical field, of EMW has the most harmful potential on living organism because of its ability to 
penetrate human bodies while electrical field has poor human skin penetration ability [5]. In fact, the induced alternating currents in our bodies resulting from cell phone EMW exposure can explain the biological non thermal effects at tissue, cellular and sub-cellular levels.

\section{Cellular and Sub-Cellular Level}

At the cellular and sub-cellular level, EMW may exert direct or indirect effects on cell membranes, cytoplasm and nucleus. Fig. (1) demonstrates some of these cellular and sub-cellular interactions with cell phone radiation.

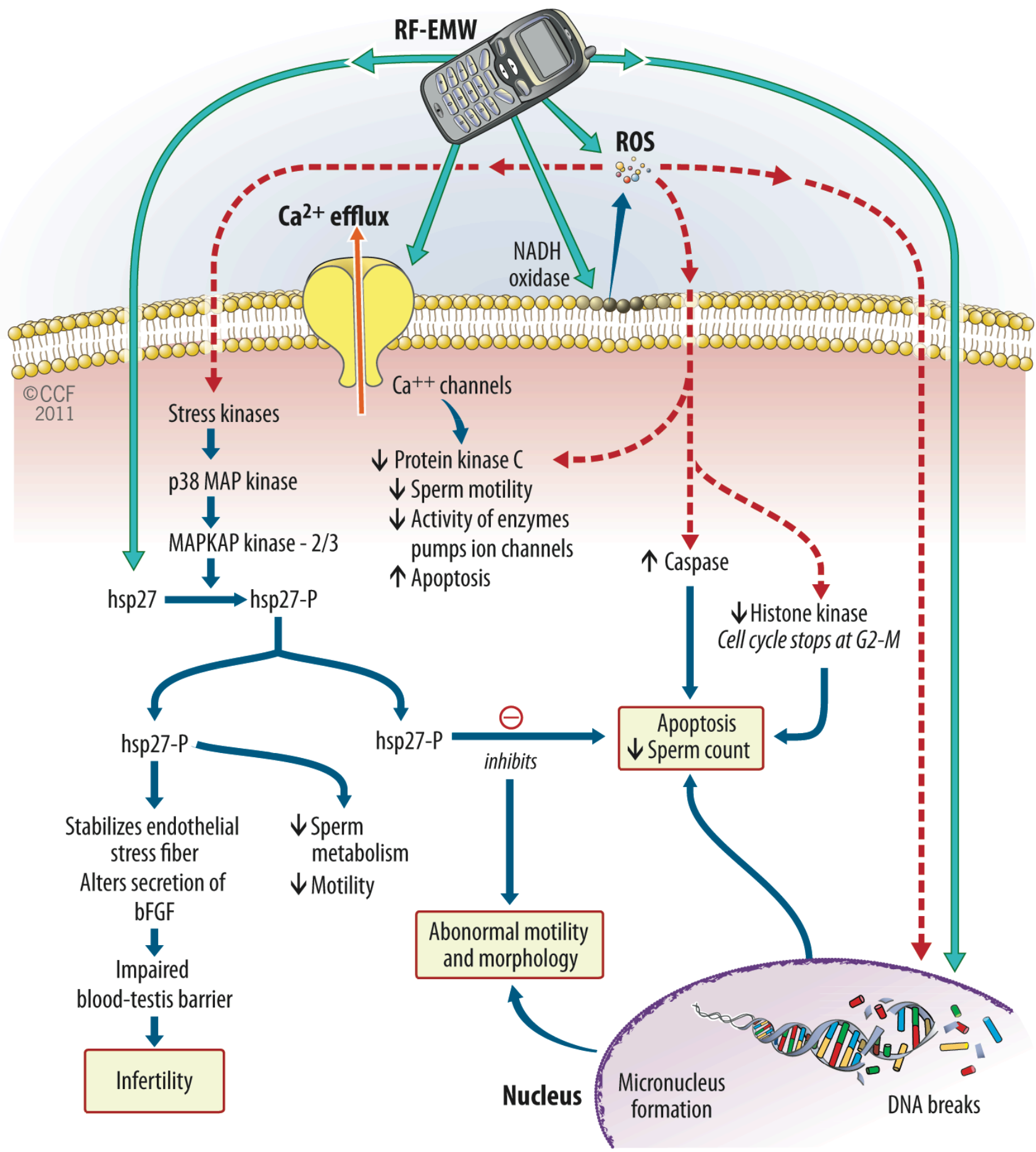

Fig. (1). Effects of RF-EMW on cellular and sub-cellular structures. Exposure to RF-EMW can induce alterations in many sub-cellular mechanisms. Changed plasma membrane potential and calcium efflux with resultant calcium depletion leads to decrease in the activity of protein kinase C (PKC). This decrease leads to alteration in many enzymes, ion pumps, channels and proteins as well as inducing apoptosis. RF-EMW also induce ROS production through disturbance of the mitochondrial membrane bound NADH oxidase. ROS has impact on PKC, histone kinase, heat shock protein, DNA and apoptosis. Heat shock protein (hsp) increases in response to electromagnetic radiation and ROS. Hsp slows the metabolism of the sperm and impairs the blood testis barrier, and interferes with apoptosis of damaged and transformed sperm. Genotoxic effect of RF-EMW on sperm is either through ROS production or through direct clastogenic chromatin breaking effect. 


\section{Plasma Membrane and Calcium Homeostasis}

In essence, all tissues are composed of cells and extracellular fluid. The intracellular fluid with cytoplasm and nucleus has similar electrical conductivity to the extracellular fluid [6].

Cell plasma membrane is negatively charged and acts to insulate the inside of the cell from the surrounding. Eddy currents formed from exposure to EMW can excite membranes causing shock and other effects. Moreover, when the external electrical field voltage across the membrane exceeds a certain threshold, large aqueous pores on the membrane are formed. This phenomenon is called electroporation [10]. This results in the plasma membrane becoming leaky with subsequent loss of intracellular molecules, ions and even macromolecules.

Meanwhile, altered calcium homeostasis due to low frequency EMW exposure leads to plasma membrane weakening. Negatively charged phospholipid bilayer of the cell membranes binds to divalent cations, such as calcium, which fit between phospholipid molecules and prevents them from repelling each other. Consequently, calcium helps to stabilize and strengthen cell membranes [11-13]. Many studies showed that under induced alternating voltage across the cell membrane, submembrane calcium ions are displaced on and off the cell membrane. Later, potassium ions, the most abundant intracellular monovalent cation, replace the sub-membrane divalent calcium ions. However, the monovalent potassium cations have a limited potential to stabilize the plasma membrane $[11,14-16]$. Therefore, the membrane becomes leaky and unstable. Additionally, electrophysiological studies on nerve cells identified the phenomenon of calcium depletion under the EMW exposure. Calcium efflux through the voltage gated calcium channels or through activation of calcium pumps can deplete the cells of calcium and consequently alter their metabolism $[11,14-16]$. Sperm are electrically active cells that depend on $\mathrm{Ca}^{2+}$ entry through calcium channels for its motility and other physiological functions. $\mathrm{Ca}^{2+}$ depletion and loss of macromolecules such as ATP from leaky plasma membranes result in poor sperm motility. Moreover, the theories of leaky plasma membrane and $\mathrm{Ca}^{2+}$ depletion may highlight the permeability problem of the blood testis barriers under EMW exposure.

It is important to realize that both plasma membrane and calcium ions play critical role in regulating the signal transduction inside the cells. As matter of fact, the plasma membrane through its integral proteins, which have intracellular catalytic enzyme activity, and through the anchored cytoskeleton controls the metabolism, gene expression and protein synthesis. On the other hand, calcium ion is a known intracellular second messenger that regulates various biological activities by signal transduction. For example, protein kinase $\mathrm{C}(\mathrm{PKC})$ is one of the key regulatory enzymes that control other activities in the cell such as cell proliferation, protein synthesis, and inhibition of apoptosis [17-19]. Meanwhile, PKC is a signally transduced protein that is activated by $\mathrm{Ca}^{2+}$ or plasma membrane proteins. PKC is localized in sperm flagellum and thus regulates sperm motility. Dysfunctional sperm PKC results in unfavorable outcomes such as poor sperm motility and even apoptosis. $\mathrm{PKC}$ also regulates vital biological processes in Leydig cells and its defect may have negative impacts such as apoptosis and impaired steroidogenesis.

In conclusion, the observed harmful outcomes in the testicular cells such as sperm, Leydig cells and Sertoli cells exposed to EMW may be explained by the leaky dysfunctional plasma membrane and the altered calcium homeostasis.

\section{The Cytoplasm}

Cytoplasm is a gel like substance that is limited by the cell membrane and holds all the organelles except the nucleus. It contains the enzymes that virtually regulate most of the metabolic activity of the cells. EMW may activate certain harmful events in the cytoplasm. In fact, EMW may result in activation of secondary intracellular lysosomes triggered by various mechanisms culminating in release of their content of hydrolytic enzymes leading to apoptosis. In addition to leaky plasma membrane and altered calcium homeostasis, another mechanism, such as the formation of free radicals and oxidative stress (OS), may come into play [20]. Mitochondria are the main site of reactive oxygen species (ROS) production at a rate proportional to EMW exposure. This has been significantly shown in studies on enhanced activity of mitochondrial NADH oxidase under short-term exposure to EMW field [21]. The excess production of ROS combined with a detectable decline in the activity of antioxidant enzymes due to EMW effect, results in oxidative stress. Oxidative stress has many devastating consequences on the cells such as lipid peroxidation, protein denaturation, and DNA damage [22]. OS not only prematurely activates lysosomal enzymes but can also directly drive the cells into apoptotic pathways [23]. Indeed, all these postulated mechanism must be examined carefully in reproductive cells such as sperm, Leydig cells and Sertoli cells which have high metabolic and proliferative activity.

Furthermore, a number of studies focused on protein alterations under pulsed EMW exposure. Changes in protein conformation in terms of folding and unfolding processes can result in either an increase or decrease in their biological activity [24]. In addition, it is hypothesized that changes in ligand binding properties of cellular proteins can affect their conformation and functions. Calcium is one of such ligands which may alter not only the conformation of the bound protein but also the neighboring proteins as well [25]. Specifically, heat shock protein 70 hsp is one of the best examples of altered protein conformation, which was examined in various studies. The exposure to pulsed EMW field was demonstrated to trigger cellular heat shock or stress response [26]. The function of heat shock proteins, in general, is to act as molecular chaperones that bind the partially damaged or denatured proteins and assist in their removal. Some studies claimed that EMW induced increase in Hsp synthesis which is responsible for implementation of heat shock response [26]. However, other studies pointed out the increase in the activity of already synthesized hsp by modification of its conformation [27]. Heat shock response antagonizes the apoptotic pathways induced by EMW exposure. This antagonism results in survival of morphologically abnormal and damaged cells previously destined for death [28]. Thus, abnormal sperm morphology seen in some studies due to cell phone may be attributed to such antagonism. 


\section{The Nucleus}

Recently, evidence for pulsed EMW induced DNA and chromatin damage emerges from multiple in vitro studies on different tissues. The genotoxic effect of EM field exposure is studied on DNA and chromatin levels. Cells with limited ability of DNA repair, such as sperm, are particularly susceptible. DNA double strand breaks, if not properly repaired, may lead to cell death (apoptosis) [29]. In the event that EM field has different ways of biological interactions with cellular components, a number of theories have been postulated to delineate the EMW induced DNA damage. Direct impact theory hypothesizes that the two DNA strands represent two cables carrying induced electrical currents in opposite directions [30]. These currents form magnetic forces around each strand in opposite directions as well, leading to progressive repelling effect between the two strands. As a result, gradual separation of the two DNA chains and consequently bending and even breaks may occur. On the other hand, indirect theory attributes DNA damage to oxidative stress through ROS. ROS have been frequently implicated in oxidative DNA damage and the increased production of 8-OH-deoxyguanosine (a marker of oxidative stress) [21].

Cell phone EM field exposure has recently been entitled to have clastogenic activity (chromatin breaking effect) [31]. This activity has been examined by micronucleus testing, traditionally used to study the genotoxic effect of environmental factors and pharmaceutical compounds on chromatin material. Microneuclei $(\mathrm{MN})$ are small bodies in the cytoplasm, found in the vicinity of the nucleus of the interphase cells. These micronuclei may originate from acentric fragments (chromosome fragments lacking a centromere) due to clastogenic mutagen, or from lagging whole chromosomes, which are unable to migrate with the rest of the chromosomes during the anaphase of cell division due to aneugenic mutagen. This test is performed on lab animals after exposing them to the cell phone EMW. Exposed bone marrow and or peripheral blood erythrocytes are harvested as the testing cells. Next, calculation of micronucleated Poly Chromatic Erythrocytes (PCE) and the ratio of PCE/NCE (normochromatic erythrocytes) in stained slides are performed. Flowcytometry has higher sensitivity and specificity than manual counting, and is used in analysis of these cells. The normal $\mathrm{PCN} / \mathrm{NCE}$ ratio is reported to be 1:1 in bone marrow. An increase in NCE signals a cytotoxic effect, whereas an increase in PCE reflects a stimulation of erythrocyte proliferative activity [32]. In addition, an increase in the micronucleated PCE in the bone marrow gives clear evidence of chromatin clastogenic insult.

A recent study by Kesari et al. has shown a significant increase $(\mathrm{P}<0.002)$ in micronucleated PCE of mobile phone exposed group $(0.67 \pm 0.15)$ as compared with control group $(1.36 \pm 0.07)$, whereas, a decrease in the ratio of PCE (polychromatic erythrocyte) to NCE (normochromatic erythrocyte) in blood cells was recorded [31]. Poor DNA integrity and chromosomal breaking may ultimately jeopardize the male reproductive function. Sperm DNA damage correlates with poor sperm count and abnormal morphology. Nuclear damage in Leydig cells is associated with apoptosis and decreased testosterone production.
Equally important is the effect of EMW on cell cycle regulatory factors in the nucleus. There are key molecules that control the transition of the cell cycle from G2 to M phase. Histone kinase is an essential molecule that exerts its effect through phosphorylation and dephosphorylation of histones. During metaphase of both meiosis and mitosis, histones in the condensed chromatin are heavily phosphorylated and then dephosphorylation takes place after the anaphase and separation of the chromosomes. Histone kinase has a Cdc2 catalytic subunit which must bind with cyclin B to form a maturation promoting factor (MAP) [33]. MAP promotes the entrance into mitosis from the G2 phase by phosphorylating multiple proteins, including histones. In highly proliferative cells such as sperm, the decreased activity of histone kinase is associated with defective progression in cell cycle and defective spermatogenesis. Kesari et al. have recently shown a statistically significant decrease in mean activity of histone kinase 1 in sperm of rats post EMW radiation (SAR $0.9 \mathrm{~W} / \mathrm{kg}$ ), as compared to the control. This decline may also indicate a decrease in $\mathrm{G} 2 / \mathrm{M}$ phase transition activity and decrease rate of proliferation [31].

\section{Tissue Level}

Tissue is composed of group of principal cells in combination with other supporting cells and substances. Various cell types displayed inconsistent results in response to EMW. Moreover, tissue types such as epithelial, muscular, connective, neural and muscular tissues differ in their rate of radiation absorption and consequently, in their interactions with EMW [6].

Body organs, on the other hand, are composed of different proportions of various tissue types with different electrical properties. Therefore, no uniform response model can applied to all tissues and organs of the body based on all these discrepancies.

\section{GENERAL BODY EFFECTS}

There has been substantial development into the understanding of how cell phone radiation may alter normal bodily functions. Many studies have looked at various body tissues reaction to the radiation exposure (Fig. 2). Alternations in the central nervous system, cardiovascular system, and localized tissue effects have been analyzed. Fluctuations in electroencephalograph (EEG) pattern, sleep pattern and neuroendrocrine functions have been observed with increased cell phone handling, along with decreased cognitive function and melatonin secretion $[34,35]$. Cell phone exposure has also been shown to increase resting blood pressure and elevate heart rate [36]. Moreover, EMW radiation may alter Leydig and Sertoli cell function, leading to decreased hormone secretion which may lead to altered cell proliferation [37]. Furthermore, frequent cell phone users described a difficulty concentrating, increased fatigue, and frequent headaches, coupled with a burning sensation near the ear and tingling or numbness of exposed tissue [38]. Therefore, it can be seen that the effects of cell phone extend beyond that of the immediate exposure range, and that these effects if compounded, can have a substantial impact on the health and wellbeing of the user. 
Central Nervous System

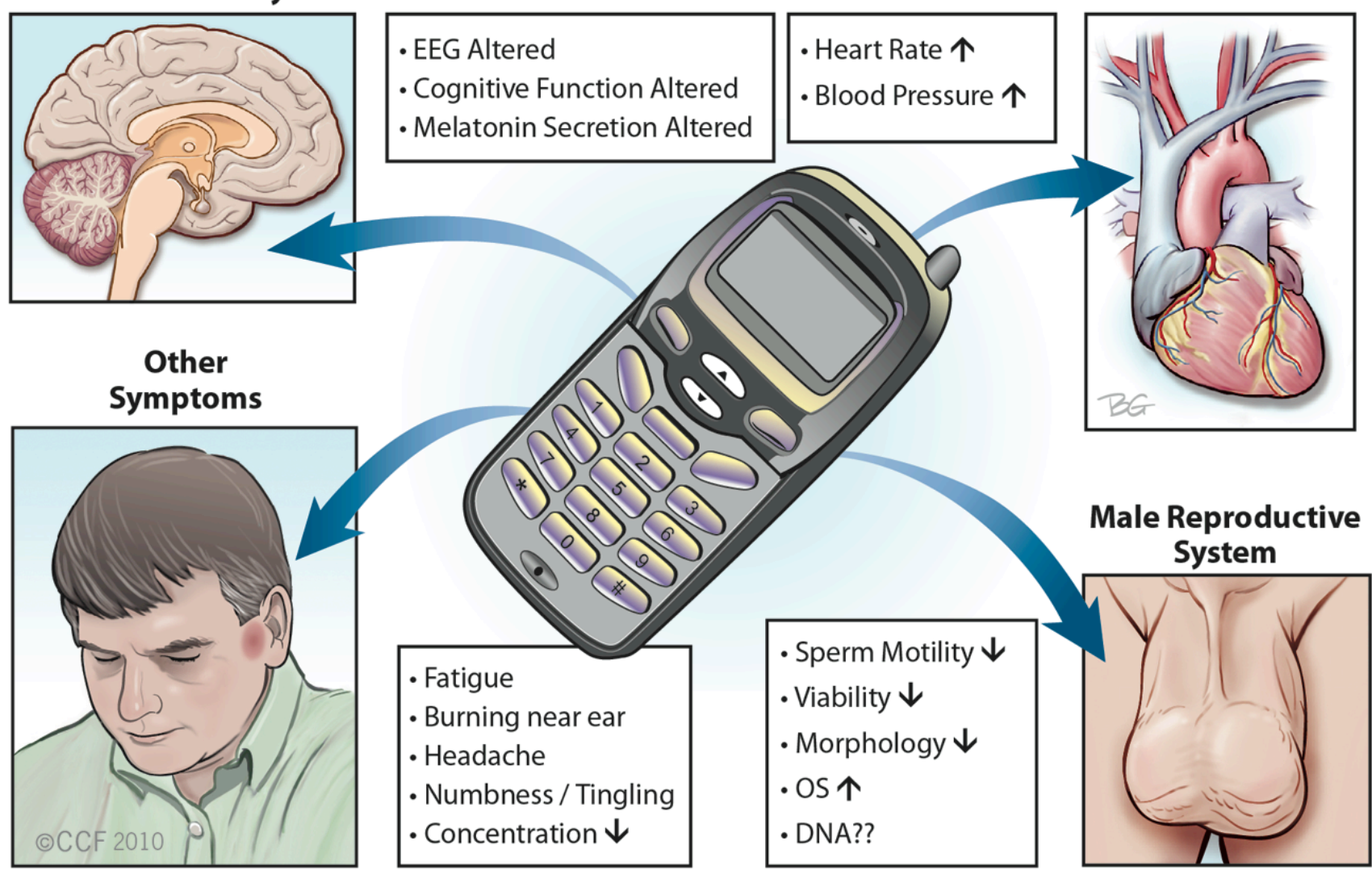

Fig. (2). Gross effects of cell phone EMW radiation.

RF-EMW emitted from Cell phones may have a detrimental effect on the cardiovascular system, central nervous system, and male reproductive system. Also, there may be generalized effects on the local tissue. Adapted from Makker 2009 [91].

\section{SEMEN AND MALE FERTILITY EFFECTS}

The frequency associated with incoming waves and subsequent energy of transmitted waves by the cellular device can have an effect on not only the general body but more specifically on the male reproductive system. Effects on sperm motility, morphology, count, sperm functions, Leydig cells, and Sertoli cells have been studied, as well as an analysis of the pituitary gland and blood-testis barrier are frequent in studies related to cell phones.

\section{Effects on Sperm}

\section{A. Sperm Motility}

There have been several studies which have looked at the detrimental effects of cell phone on the motility characteristcs of spermatozoa. Wdowiak et al. performed a retrospective study (304 men) noting that there was a significant decrease in the percentage of forward progressive motile sperm, correlated with the frequency of cell phone handling. In their study, $65.7 \%$ of patients who did not use cell phones had normal motility (over $50 \%$ of sperm with forward progressive motility) compared to only $17 \%$ of patients who frequently (regular phone use for more than 2 years) used cell phones [39].

Furthermore, in another retrospective study involving 371 men in reproductive age, the duration of using cell phone usage and the daily transmission time correlated negatively with the proportion of rapid progressive motile sperm $(r=-0.12$ and $r=-0.19$, respectively), and shared a significantly positive correlation with the proportion of slowly progressive motile sperm $(\mathrm{r}=0.12$ and $\mathrm{r}=0.28$, respectively) [40].

Also, in a study of 22 men with a mean age of 24 years, it was found that sperm samples exposed to high intensity EMW radiation $(1.8 \mathrm{GHz} \times 0.4 \mathrm{~W} / \mathrm{kg}-27.5 \mathrm{~W} / \mathrm{kg} \mathrm{SAR})$ experienced a statistically significant decline in both motility and viability. Also noted, was a significant increase in the rate of mitochondrial ROS production and the level of 8OHdG. Although the intensity of the EMW used was extremely high, this study showed that EMW radiation has the potential to cause damage not only at the morphological level but also at the molecular DNA level [21].

Moreover, Agarwal et al. conducted a prospective in vitro study of 32 men, exposing the neat semen samples to RF-EMW radiation (1.46 W/kg SAR x $60 \mathrm{~min})$. The authors noted a significant decrease in sperm motility and viability post exposure. Also observed was an increase in reactive oxygen species levels paired with a decrease in ROS-TAC score. It was concluded that RF-EMW emitted from cell phones may increase oxidative stress in human spermatozoa leading to decreased motility and viability characteristics [41]. 


\section{B. Sperm Morphology and Count}

Exposure to cell phone RF-EMW radiation has been also correlated with a decrease in normal sperm morphology and count. An observational study of 361 men was conducted to determine whether there is a correlation between cell phone usage and sperm morphology. Men were divided into four usage groups: no use, $<2 \mathrm{~h} /$ day, $2-4 \mathrm{~h} /$ day, and $>4 \mathrm{~h} /$ day. It was found a statistically significant difference in mean WHO normal morphology between the low usage group (40.32 \pm 13.06) and the high usage group (18.40 \pm 10.38$)$ [42].

Wdowiak et al. also noted a significant increase in the percentage of sperm cells with abnormal morphology correlated with the frequency and duration of exposure to cellular phone RF-EMW emitted by GSM cell phones. $55.6 \%$ of patients without cell phones had normal morphology parameters (over $30 \%$ normal shaped sperm morpho$\operatorname{logy}$, whereas only $16.7 \%$ of patients who frequently (regular phone use for more than 2 years) used cell phones had normal sperm morphology [43].

Fejes et al. showed in an observational study that a significant decrease in sperm count related to cell phone handling frequency. This study analyzed 231 men over a 13month period, and showed that for heavy users of cell phones, sperm counts were $30 \%$ lower than men who did not use a cell phone [40].

Moreover, in an animal study exposed rats to cell phone RF-EMW ( $2 \mathrm{~h} /$ day x 35 days at 0.9 SAR), showed a decreased mean value of total sperm count $(31.14 \pm 13.6 \mathrm{vs}$. $61.33 \pm 3.68)$, and an increased mean percentage of apoptotic cells $(13.15 \pm 1.26$ vs. $5.93 \pm 1.64 \%)$ [44]. Furthermore, Salama et al. conducted a study on rabbits exposed to mobile phone radiation (GSM mode, $800 \mathrm{MHz}$, standby status). RF-EMW exposure of 8 hours/day led to a significant decline in the sperm count after 8 weeks of exposure and a decrease in motility after 10 weeks of exposure [45]. In contrast, other studies did not show a correlation between cell phone EMW radiation and alteration in sperm count.

\section{Sperm Function}

\section{$\underline{\text { ROS Testing and TAC }}$}

ROS have been implicated as the fundamental mechanism of cell phone EMW damage on sperm. Sperm are efficient ROS producer because ROS are needed to perform many physiological functions. At the same time, sperm are vulnerable to the effect of ROS because of limited reserve of cytoplasmic antioxidants and their high membrane content of polyunsaturated fatty acids (PUFA) [46]. Oxidative stress is said to be present whenever there is an excess of ROS or decrease in total antioxidant capacity (TAC).

Admittedly, it is well known that OS has harmful consequence on male reproductive function and semen parameters as well as sperm function.

Originally, Grundler et al. were the first to demonstrate that EMW induce free radical activity in cells [47]. Animal studies have shown various examples of cell phone induced $\mathrm{OS}$ in the eyes, brain, kidneys and endometrial lining of uterus [48-51].
De Iuliis et al. showed increased sperm mitochiondrial ROS production as mentioned above. The authors reported that with increase in SAR, the sperm motility and vitality are decreasing while mitochondrial production of ROS and DNA integrity defects using TUNEL are increasing $(\mathrm{p}<$ 0.001) [21] Similarly, Agarwal et al. demonstrated an increase in ROS levels coupled with a decrease in ROS-TAC score [52].

Most recently, Kesari et al. have shown a significant increase in ROS levels in the semen of male rats exposed to mobile phones $(58.25 \pm 10.36 \mathrm{mg} / \mathrm{l})$ as compared to the semen of sham exposed animals $(41.78 \pm 12.93 \mathrm{mg} / \mathrm{l}) \quad(\mathrm{P}=0.035)$ [31].

However, another study failed to find any significant increase in ROS level in sperm post cell phone EMW exposure. This study used purified leukocyte free sperm, which may be the reason for not getting excess ROS since leukocytes are a major source of ROS production in semen [53].

Moreover, a decrease in total antioxidant activity has been examined in other studies [28, 31, 54,55]. Moustafa et al. demonstrated a decrease in the activity of antioxidant enzymes (such as superoxide dismutase and glutathione peroxidase) in erythrocytes in humans exposed to RF-EMW [56]. Chronic exposure to RF-EMW decreases the activity of catalase, superoxide dismutase (SOD) and glutathione peroxidase (GSH-Px), and thus decreases the total antioxidant capacity in different organs of the body [45, 57-60]. A decrease in the level of SOD activity suggests an increase in the generation of reactive superoxide ions [61].

Regarding seminal level of antioxidant enzymes, Kesari et al. confirmed a decrease in GSH-Px and SOD and an increase in catalase levels post cell phone EMW radiation (SAR $0.9 \mathrm{~W} / \mathrm{kg}$ ) in a study on mice testes [31].

Studies have also demonstrated that antioxidants such as melatonin, caffeic acid, phenyl ester, vitamin $\mathrm{C}$, and vitamin E prevent oxidative stress or apoptosis caused by RF-EMW in different animal tissues $[51,55,57]$. Likewise, melatonin is a hormone secreted from the pineal gland and acts as a natural antioxidant, protecting against lipid peroxidation in the retina, brain, liver and human sperm [62]. There has also been a demonstrated reduction in 6-hydroxymelatonin sulfate (6-OHMS) levels in the urine among individuals using a cell phone for over 25 minutes/day [63]. 6-OMHS is the urinary metabolite reflecting the serum level of the pineal hormone melatonin [62]. Therefore, exposure to cell phones may be correlated with significant decrease of melatonin in the body, making spermatozoa more susceptible to reactive oxygen species attack. Thus, the effect of cell phone EMW on generation of oxidative stress cannot be ignored based on the above evidence. The consequence of such ignorance may be dismal in regards to male infertility in the future.

\section{DNA Integrity Defects}

Deoxyribonucleic acid (DNA) contains all the genetic information that controls the vital functions of the sperm. DNA damage from external and internal sources is correlated with poor semen quality and poor fertilization rate, as well as poor pregnancy outcomes [64]. Sperm have limited 
ability to repair single or double strand DNA breaks. DNA damage and chromatin breakage effects have been demonstrated as one of the biological interactions of cell phone EMW in different tissues.

A number of studies have been performed to examine the DNA damaging effect of cell phone EMW on sperm. Aitken et al. reported that a 7-day exposure of male mice to RF EMFs induced significant DNA damage in both the nuclear and mitochondrial genomes of spermatozoa retrieved from the cauda epididymis [65]. De Iuliis have shown the increment of sperm DNA integrity defects by TUNEL assay under cell phone EMW effect is proportional to the exposed SAR [21].

In contrast, Falzone et al. had used TUNEL assay and did not find any significant DNA integrity defect in the examined purified sperm under EWM exposure [53]. Similarly other study by Agarwal failed to show any difference with regard to EMW impact on in vitro sperm [52].

The important conclusion drawn from the above studies is that DNA damage due to EMW is significant. However, this damage may be of cumulative effect of repeated exposure and it may not be revealed after short term exposure. Also, the difference in the reported results from the above mentioned studies may be attributed to differences in the frequency of applied EMW and SAR.

\section{Tests for Capacitation and Fertilization}

Sperm function in terms of capacitation and fertilization has not been thoroughly studied in the literature under cell phone electromagnetic wave exposure. However, Falzone et al. reported that at a SAR of $5 \mathrm{~W} / \mathrm{kg}$, hyperactivation (HA) motility parameters are impaired, particularly straight line velocity (VSL) and beat cross frequency (BCF) [66]. This defect in HA may lead to poor fertilization rates. However, the SAR used in this experiment was higher than the traditional SAR of modern cell phones. Another observed finding was the absence of thermal influence as the rise in temperature was only $0.38 \mathrm{C}^{\circ}[66]$. The effect on HA may be elucidated through effect of EMW on ROS, DNA integrity defects or $\mathrm{Ca}^{2+}$ depletion.

Furthermore, another in vitro study on purified human sperm by Falzone et al. failed to identify any difference in premature acrosome reaction due to cell phone exposure (900 MHz SAR $2 \mathrm{~W} / \mathrm{kg}$ x 1 hour). In contrast, the authors observed significant decrease in sperm zona pellucida binding which certainly correlated with poor fertilization rate [67].

\section{Effects on Leydig Cells}

Leydig cells, also known as interstitial cells of the testis, are responsible for secretion of androgen under the effect of Luteinizing Hormone. In fact, Leydig cells account for $75 \%$ of total body testosterone [68]. Testosterone secretion is essential for initiation and maintenance of spermatogenesis. Generally, Leydig cells are known for their resistance to the effects of ionizing radiation across a wide range of exposed doses [68]. However, the impact of EMW on the function of Leydig cells has been examined in a variety of animal studies. The results of these studies are still conflicting.
In a study on mice Wang et al. demonstrated that Leydig cells are among the most susceptible cells to EMW and that injury to these cells may affect spermatogenesis [69]. Oxidative stress and EMW induced alteration in PKC enzyme complex, which is present in seminiferous tubules and leydig cells, can explain the deranged function of leydig cells in response to cell phone exposure.

Moreover, Wang et al. discovered a decrease in serum testosterone in the exposed group in comparison to the controls [70]. Additionally, Zhou et al. confirmed that EMW not only alters serum testosterone but also affects the expression of mRNA for P450 cholesterol side chain lyase (the first enzyme in steroidogenesis) in Leydig cells [70]. Again, another study on rats showed that serum testosterone was significantly reduced in the EMW group compared to control group $(p<0.05)$ although there was no demonstrable changes in FSH, LH, or interstitial histology. The study concluded that the normal FSH and LH levels may be explained by minimal exposure of EMW on the anterior pituitary which leads to inhibition of excessive $\mathrm{FSH}$ and $\mathrm{LH}$ release in response to low testosterone [71].

Equally important, Salama et al. measured androgendependent secretory activity of accessory sex glands in rabbits exposed to cell phone radiation and discovered a significant decline in seminal plasma fructose in the exposed group after 10 weeks of exposure [72]. However, the authors did not find any difference in serum testosterone levels between the study groups. The authors attributed these findings to possible alteration in testosterone receptors or oxidative stress on male accessory glands [72]. On the other hand, the EMW impact Leydig cells not only through functional alterations, but also through structural and pathological changes. A study by Wang et al., detected pathological changes such as edema and vacuolation, swelling of cytoplasmic mitochondria, reduction of lipid droplets, pale staining of most of lipid droplets, and partial or complete cavitation of lipid droplets in Leydig cells within 28 days after EMW radiation [70].

In contrast, post two weeks exposure, Forgacs et al. found an increase in testosterone level [73]. Moreover, Khillare et al. found no demonstrable alteration in serum testosterone in male rats exposed to $200 \mathrm{MHz} \mathrm{RF}$ fields, (SAR 2.0-1.65 W/kg) for 35 days of exposure $(2 \mathrm{~h} /$ day x 6 day/week) [74]. However, the authors detected some ultrastructural changes in Leydig cells. Correspondingly, further animal studies failed to detect significant alterations in testosterone level or histological alterations in leydig cells in response to cell phone EMW [45, 58, 75].

Recently, Kesari and Behari have reported increased apoptosis in Leydig cells of testis due to microwave exposure at $2.45 \mathrm{GHz}$ and $0.11 \mathrm{~W} / \mathrm{kg}$ of SAR on 35 days of exposure [76]. Elevated apoptotic rate in this study may be explained by various mechanisms as have been described under the subject of biological interactions.

In conclusion, analysis of the above information shows the impact of cell phones on spermatozoa and associated tissues is seemingly real. Nevertheless, it is not known whether the damage is reversible and temporary or it is irreversible, giving the fact that there are no demonstrable 
pathological changes at the current cell phone radiation. The conflicts in the various results may emerge from different study design and different EMW application doses and duration. Further research in this field is certainly needed to explore the damage based on standardized study protocol and graded response measurement.

\section{Effects on Sertoli Cells and Blood-Testis Barrier}

Blood testis barrier (BTB) is the physical barrier that separates blood and lymph vessels from seminiferous tubules. Histologically, it is formed of tight junctions between Sertoli cells. The main function of BTB is protecting the newly developing sperm from immunological recognition by the body's immune system, in addition to prevention of leaking of testicular immunogenic materials.

Interestingly, the known damaging effect of cell phone EMW on blood brain barrier raises the concern about the effect on the integrity of BTB as well [77]. There are two studies which examined the effects of EMW on BTB integrity. However, both studies used higher electrical field intensity than that of current cell phones. A recent study by Wang et al. has been conducted on mice testes, exposed to two different EF intensities 200 and $400 \mathrm{kv} / \mathrm{m}$ respectively [77]. Analysis of the results revealed morphological alterations of Sertoli cells represented by cellular swelling with nuclei being circular or oval situated in the center rather than basally. The number of cellular processes increased and were closely interconnected with observed numerous cytoplasmic granules and vacuoles. Although Sertoli content of vimentin, an intermediate filament that constitute the cytoskeleton, showed no difference in this study, the length of vimentin filaments showed progressive shortening after 1 to 2 hours of exposure, with restoration of the original length after 6 hours. Certainly, the alterations in the arrangement of vimentin filaments affect the Sertoli cell morphological shape and the integrity of its tight junctions and hence the BTB [78].

There are four important Sertoli cell integral membrane tight junction proteins that regulate the BTB. These proteins are: occludins, claudins, ZO-1, and JAM (junctional adhesion molecules) [79-81]. The above study measured the content of ZO-1 and occludin in Sertoli cells tight junctions and found a decline in the expression of ZO-1 but not occludin. $\mathrm{ZO}-1$ is an intracytoplasmic protein that combines the tight junction membranes protein occludin and the cyto-skeleton [78].

Occludin is an integral membrane protein with an intracellular component that depends on $\mathrm{Ca}^{2+}$ and performs defensive and barrier functions. Although the content of this protein did not change in this study, the altered calcium homeostasis presented earlier in the biological interactions could interfere with its function. Moreover, transforming growth factor beta $\left(\mathrm{TGF}_{\mathrm{B}}\right)$ was also measured because it regulates the Sertoli cell kinetics and decreases the expression of the tight junction protein $\mathrm{ZO}-1$. The study noticed an increase in the expression of mRNA of TGFB [78]. All these deranged parameters in Sertoli cells can be explained on the basis of free radical generation theory under EMW exposure and calcium depletion effect as well as the EMW induced membrane electroporation. The same study found that $90 \%$ of the exposed group have detectable serum antisperm antibody while none in the controls indicated an increase in the permeability of BTB [78].

Another study used two different types of tracers such as Evans Blue and Lanthanum nitrate to investigate the integrity of mice BTB after EMW exposure $(200 \mathrm{kv} / \mathrm{m})$. The authors found the tracers were able to penetrate BTB and reach to the seminiferous tubules indicating damage [82].

Despite higher electrical fields used in these studies, they raise the level of understanding against a further increase in current cell phone EMW technology. This increase may be severely detrimental to the structural supporting components of the testis.

Fig. (3) shows the pathological effects of cell phone on various cellular components of testes.

\section{Effects on Pituitary Gland}

The pituitary gland secretes gonadotropins FSH and LH that regulate testicular spermatogenesis and steroidogenesis. Effect of EMW on gonadotropins level has been studied in humans and animal models. De Seze et al. examined the gonadotropins concentrations of anterior pituitary hormones FSH and LH in 21 healthy males after applying $900 \mathrm{MHz}$ RF radiation emitted from a cell phone $(2 \mathrm{~h} /$ day x 5 days/week $\mathrm{x}$ 1 month) and found no effect [83]. However, the duration of $\mathrm{RF}$ radiation exposure in their study might not be sufficient to produce any significant effect. Other studies also failed to prove pituitary gonadotropins alteration in humans or animals exposed to cell phone $[73,84,85]$.

On the other hand, a recent report by Fang et al. showed progressive histological derangement in rats' pituitary glands exposed to high level of EMW $(200 \mathrm{kv} / \mathrm{m})$ in the form of swollen mitochondria as well as dilatation of Golgi complex and diffusive lysosomes. With increasing duration of exposure and EMW energy, mitochondrial vacuolization, formation of myelin figures, distinct dilatation of endoplasmic reticulum, occurrence of numerous secondary lysosomes, and clustering of heterochromatin under the nuclear membranes could be observed [86].

On the whole no study showed that the present level of energy of cell phone EMW has detrimental effect on pituitary secretion of gonadotropins. Despite the fact that the level of electrical field strength used in the above animal study is higher than the current level found in modern cell phones, it should be noted that this level of radiation may cause substantial damage. Therefore, every effort should be undertaken to prevent any further increase in the radiation and electrical strength levels found in today's cell phones.

\section{AMBIGUITY SURROUNDING CELL PHONE STUDIES AND THEIR RESULTS}

The field of cell phone research, and RF-EMW radiation affecting human health, is surrounded by controversy. As shown, there have been many studies which point toward a negative correlation between cell phone and both gross human health and male reproduction. However, there are equally as many studies which conclude that the effects of RF-EMW are negligible and that there is no correlation between cell phones and semen parameters [40, 45, 60, 87]. 


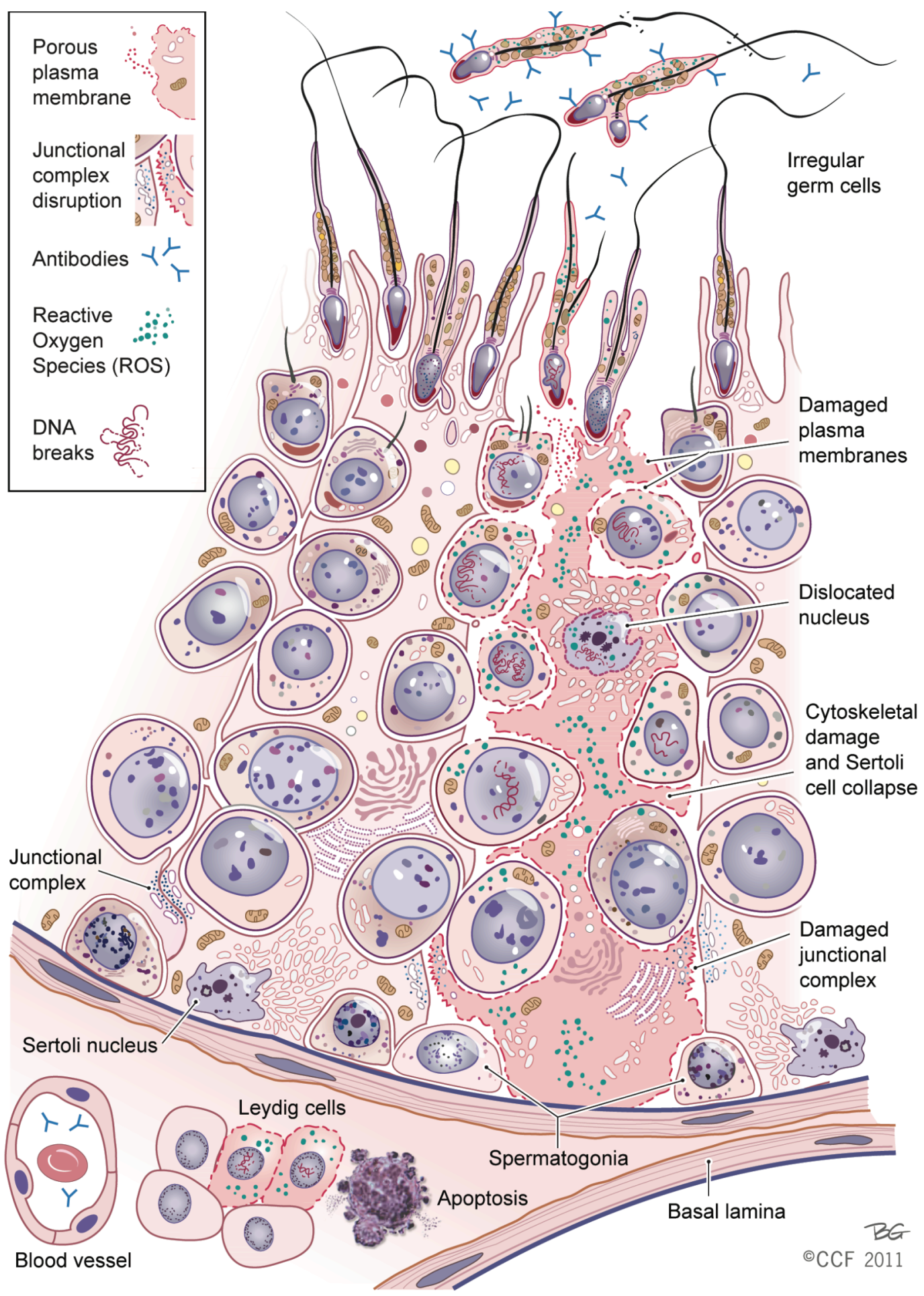

Fig. (3). Cross sectional view of testicular tissue showing various effects of cell phone RF-EMW on cellular components of the testis. In sperm: a) plasma membrane becomes leaky and porous due to EMW induced electroporation, b) cytoplasmic mitochondria generate excess ROS resulting in oxidative stress, c) nuclear DNA and chromatin undergo breaks and damage. In Sertoli cells: a) damage to plasma membrane tight junctional complexes compromises the integrity of BTB and increases its permeability resulting in exposure of sperm antigens to immune system and formation of ASA, b) damage to cytoskeleton results in cell collapse with c) production of excess ROS, and d) dislocation of nucleus to a more central position. In Leydig cells: a) plasma membrane sustains damage with b) excessive cytoplasmic ROS generation, and c) nuclear DNA damage resulting in apoptosis.

Abbreviations: RF-EMW (radiofrequency-electromagnetic wave), ROS (reactive oxygen species), DNA (deoxyribonucleic acid), BTB (blood-testis barrier), ASA (antisperm antibody)."

Controversy in this field stems from inadequate study design, ethics, and biological variability between individuals.
Appropriate, repeatable study design is hard to attain due to a variety of factors including finding an adequate control 
group, variations in sperm cell types used, and testing protocol in terms of wave frequencies and duration of exposure. The appropriate control group in a cell phone trail must be an individual who has not had any previous exposure to any form of cellular device. This, however, is hard to attain due to the technological growth occurring every day. Therefore, individuals who have "limited" exposure to cell phone RFEMW radiation are used as a standard for in vivo testing.

Another flaw in cell phone studies is a non-standardized testing protocol. There is no assay highlighting specific sperm cell stage, to study the maturity of these cells and methods of preparing and exposing sperm. Also, there is a lot of variation in terms of the frequency of the radio waves, the SAR at which the phone runs, and the duration of exposure to the cells. Furthermore, there are differences in the type of cellular device used, the transmission mode at which it operates (talk vs. standby) and also the distance between the cells and the phone. All of these variations contribute to the ambiguity of the results present in cell phone studies.

Moreover, the measured SAR varies in studies due to several parameters including frequency, intensity, polarization, and radiation source-body configuration. The exposure also depends on the cell types, shape, location, size, and electrical properties of the body. Also, animals have different body sizes and reproductive tract difference in term of anatomy characteristics from human, which make the application of animal study results to humans seemingly arguable.

In fact, not all the exposed men will be infertile. This notion arises from observation of inconsistent reactions of various human body system of diverse group of people to weak electromagnetic radiation and ionizing radiation. It has been estimated that $3 \%$ of people are considered electrosensitive because they experienced a wide range of unpleasant vague symptoms such as nervousness, headache and other behavioral problems when exposed to weak nonionizing radiation. Contrastingly, other individuals who are electrosensitive do not realize their problems when they are continuously exposed to this type of radiation and they report their symptoms as being perfectly normal [88]. Theories behind electrosensitivty include presence of thicker layer of stratum granulosum in their skin which is regarded as the leaky layer [89]. Consequently, the total energy and SAR delivered to superficial organs such as the testis will be more. Others attribute this undue sensitivity to relatively low blood calcium and magnesium. Such low levels entail that fewer ions are needed to move across the plasma membranes under EMW exposure, causing noticeable disturbance in the cells and tissues [88].

Lastly, people differ in their inherited sperm characteristics such as DNA repair system and antioxidant capacity, which are the main defense mechanisms against cell phone EMW radiation.

For the ethical point of view, specific considerations must be taken into account when conducting in vivo testing. For this reason most trials are conducted either in animal models or in vitro ejaculated samples. The inherent problems associated with using animal models have previously been elucidated. The complications that arise when conducting in vitro testing with ejaculated neat samples is determining the distance between the exposure device and the sample in order to mimic real life conditions. To solve this problem the Finite Difference Time Domain (FDTD) method was used. This computer-assisted simulation mimicked the effects of multiple tissue layers between a cell phone and the spermatozoa in the testis. The results indicated that in order to simulate in vivo exposure, the distance between a cell phone and an ejaculated semen sample should be $0.8 \mathrm{~cm}$ to $1.8 \mathrm{~cm}$ greater than the anticipated distance between the cell phone and the testis [90]. These results can be used to construct better-designed studies in the future.

No significant conclusions regarding cellular phone RFEMW radiation effects on the human body and the male reproductive system can be drawn until there is a formalized study protocol for cell phone RF-EMW exposure that takes into account an adequate control group and limits external design variations.

\section{ACKNOWLEDGEMENTS}

None Declared.

\section{CONFLICT OF INTEREST}

None Declared.

\section{REFRENCES}

[1] Brown T. Historical first patents: the first United States patent for many everyday things. Metuchen. NJ Scarecrow Press 1994.

[2] Digital Wireless Basics: Frequencies V Cellular, PCS, GSM, and Japanese Digital Cellular Frequencies. 2007. (Accessed at www. privateline.com/PCS/Frequencies.htm).

[3] How cell-phone radiation works. 2007. (Accessed at electronics. how.stuffworks.com/cell-phone-radiation.htm/printable).

[4] Roelandts R. Cellular phones and the skin. Dermatology 2003; 207: 3-5.

[5] Cleveland RFS, David MU, Jerry L. Evaluating Compliance with FCC Guidelines for Human Exposure to Radiofrequency Electromagnetic Fields. 1997; OST Bulletin No. 65 ed. 97-01.

[6] Habash RWY. Bioeffects and therapeutic applications of electromagnetic energy. Boca Raton: CRC Press 2008.

[7] Institute of Electrical and Electronics Engineers. Proceedings of the IEEE. Institute of Electrical and Electronics Engineers, New York 1963.

[8] Inan US, Inan AS. Engineering electromagnetics. Menlo Park, Calif: Addison-Wesley 1999.

[9] Deepinder F, Makker K, Agarwal A. Cell phones and male infertility: dissecting the relationship. Reprod Biomed Online 2007; 15: 266-70

[10] Neumann E, Schaefer-Ridder M, Wang Y, Hofschneider PH. Gene transfer into mouse lyoma cells by electroporation in high electric fields. EMBO J 1982; 1: 841-5.

[11] Ha BY. Stabilization and destabilization of cell membranes by multivalent ions. Phys Rev E Stat Nonlin Soft Matter Phys 2001; (64) 051902: 1-5.

[12] Lew VL, Hockaday A, Freeman CJ, Bookchin RM. Mechanism of spontaneous inside-out vesiculation of red cell membranes. J Cell Biol 1988; 106: 1893-901.

[13] Steck TL, Weinstein RS, Straus JH, Wallach DF. Inside-out red cell membrane vesicles: preparation and purification. Science 1970; 168: 255-7.

[14] Bawin SM, Kaczmarek LK, Adey WR. Effects of modulated VHF fields on the central nervous system. Ann N Y Acad Sci 1975; 247: 74-81.

[15] Blackman CF, Benane SG, House DE, Elliott DJ. Importance of alignment between local DC magnetic field and an oscillating 
magnetic field in responses of brain tissue in vitro and in vivo. Bioelectromagnetics 1990; 11: 159-67.

[16] Blackman CF, Benane SG, Kinney LS, Joines WT, House DE. Effects of ELF fields on calcium-ion efflux from brain tissue in vitro. Radiat Res 1982; 92: 510-20.

[17] Larsson C. New insights into PKC family affairs: three novel phosphorylation sites in PKCepsilon and at least one is regulated by PKCalpha. Biochem J 2008; 411: 15-6.

[18] Kimura K, Katoh N, Sakurada K, Kubo S. Phospholipid-sensitive $\mathrm{Ca}^{2+}$-dependent protein kinase system in testis: localization and endogenous substrates. Endocrinology 1984; 115: 2391-9.

[19] Naor Z, Breitbart H. Protein kinase C and mammalian spermatozoa acrosome reaction. Trends Endocrinol Metab 1997; 8: 337-42.

[20] Panagopoulos DJ. Analyzing the Health Impacts of Modern Telecomumications Microwaves. Adv Med Biol 2011; 17: 1-55

[21] De Iuliis GN, Newey RJ, King BV, Aitken RJ. Mobile phone radiation induces reactive oxygen species production and DNA damage in human spermatozoa in vitro. PLoS One 2009; 4(7): e6446.

[22] Barzilai A, Yamamoto K. DNA damage responses to oxidative stress. DNA Repair (Amst) 2004; 3: 1109-15.

[23] Simon HU, Haj-Yehia A, Levi-Schaffer F. Role of reactive oxygen species (ROS) in apoptosis induction. Apoptosis 2000; 5: 415-8.

[24] Bohr H, Bohr J. Microwave enhanced kinetics observed in ORD studies of a protein. Bioelectromagnetics 2000; 21: 68-72.

[25] Chiabrera A, Bianco B, Moggia E, Kaufman JJ. Zeeman-Stark modeling of the RF EMF interaction with ligand binding. Bioelectromagnetics 2000; 21:312-24.

[26] Blank MGR. Stimulation of the stress response by low-frequency electromagnetic fields: possibility of direct interaction with DNA. IEEE Trans Plas Sci 2000; 28: 168-72

[27] Laurence JA, French PW, Lindner RA, McKenzie DR. Biological effects of electromagnetic fields--mechanisms for the effects of pulsed microwave radiation on protein conformation. J Theor Biol 2000; 206: 291-8.

[28] Desai NR, Kesari KK, Agarwal A. Pathophysiology of cell phone radiation: oxidative stress and carcinogenesis with focus on male reproductive system. Reprod Biol Endocrinol 2009; 7: 114-123.

[29] Lai H, Singh NP. Single- and double-strand DNA breaks in rat brain cells after acute exposure to radiofrequency electromagnetic radiation. Int J Radiat Biol 1996; 69: 513-21.

[30] Blank M, Goodman R. Electromagnetic fields may act directly on DNA. J Cell Biochem 1999; 75: 369-74.

[31] Kesari KK, Kumar S, Behari J. Effects of radiofrequency electromagnetic wave exposure from cellular phones on the reproductive pattern in male wistar rats. Appl Biochem Biotechnol 2011; 164(4):546-59

[32] Gollapudi BB, McFadden LG. Sample size for the estimation of polychromatic to normochromatic erythrocyte ratio in the bone marrow micronucleus test. Mutat Res 1995; 347: 97-9.

[33] Gautier J MJL. Cyclin B in Xenopus oocytes: implications for the mechanism of pre-MPF activation. EMBO J 1991; 10: 177-82.

[34] D'Costa H, Trueman G, Tang L, et al. Human brain wave activity during exposure to radiofrequency field emissions from mobile phones. Australas Phys Eng Sci Med 2003; 26: 162-7.

[35] Kramarenko AV, Tan U. Effects of high-frequency electromagnetic fields on human EEG: a brain mapping study. Int J Neurosci 2003; 113: 1007-19.

[36] Braune S, Wrocklage C, Raczek J, Gailus T, Lucking CH. Resting blood pressure increase during exposure to a radio-frequency electromagnetic field. Lancet 1998; 351: 1857-8.

[37] Roosli M, Michel G, Kuehni CE, Spoerri A. Cellular telephone use and time trends in brain tumour mortality in Switzerland from 1969 to 2002. Eur J Cancer Prev 2007; 16: 77-82.

[38] Oftedal G, Wilen J, Sandstrom M, Mild KH. Symptoms experienced in connection with mobile phone use. Occup Med (Lond) 2000; 50: 237-45.

[39] Wdowiak A, Wdowiak L, Wiktor H. Evaluation of the effect of using mobile phones on male fertility. Ann Agric Environ Med 2007; 14: 169-72.

[40] Fejes I, Zavaczki Z, Szollosi J, et al. Is there a relationship between cell phone use and semen quality. Arch Androl 2005; 51: 385-93.

[41] Agarwal A, Desai NR, Makker K, et al. Effects of radiofrequency electromagnetic waves (RF-EMW) from cellular phones on human ejaculated semen: an in vitro pilot study. Fertil Steril 2009; 92: 1318-25.
[42] Agarwal A, Deepinder F, Sharma RK, Ranga G, Li J. Effect of cell phone usage on semen analysis in men attending infertility clinic: an observational study. Fertil Steril 2008; 89: 124-8.

[43] Wdowiak A, Wdowiak L, Wiktor H. Evaluation of the effect of using mobile phones on male fertility. Ann Agric Environ Med 2007; 14: 169-72.

[44] Kesari KK, Kumar S, Behari J. Mobile phone usage and male infertility in Wistar rats. Indian J Exp Biol 2010; 48: 987-92.

[45] Salama N, Kishimoto T, Kanayama HO. Effects of exposure to a mobile phone on testicular function and structure in adult rabbit. Int J Androl 2010; 33: 88-94.

[46] Aitken RJ, Baker MA. Oxidative stress, sperm survival and fertility control. Mol Cell Endocrinol 2006; 250: 66-9.

[47] Grundler W, Kaiser F, Keilmann F, Walleczek J. Mechanisms of electromagnetic interaction with cellular systems. Naturwissenschaften 1992; 79: 551-9.

[48] Meral I, Mert H, Mert N, et al. Effects of 900-MHz electromagnetic field emitted from cellular phone on brain oxidative stress and some vitamin levels of guinea pigs. Brain Res 2007; 1169: 120-4.

[49] Balci M, Devrim E, Durak I. Effects of mobile phones on oxidant/antioxidant balance in cornea and lens of rats. Curr Eye Res 2007; 32: 21-5.

[50] Ozguner F, Bardak Y, Comlekci S. Protective effects of melatonin and caffeic acid phenethyl ester against retinal oxidative stress in long-term use of mobile phone: a comparative study. Mol Cell Biochem 2006; 282: 83-8.

[51] Oktem F, Ozguner F, Mollaoglu H, Koyu A, Uz E. Oxidative damage in the kidney induced by $900-\mathrm{MHz}$-emitted mobile phone: protection by melatonin. Arch Med Res 2005; 36: 350-5.

[52] Agarwal A, Desai NR, Makker K, et al. Effects of radiofrequency electromagnetic waves (RF-EMW) from cellular phones on human ejaculated semen: an in vitro pilot study. Fertil Steril 2009; 92: 1318-25.

[53] Falzone N, Huyser C, Franken DR, Leszczynski D. Mobile phone radiation does not induce pro-apoptosis effects in human spermatozoa. Radiat Res 2010; 174: 169-76.

[54] Kumar S, Kesari KK, Behari J. Evaluation of genotoxic effects in male Wistar rats following microwave exposure. Indian J Exp Biol 2010; 48: 586-92.

[55] Kumar S, Kesari KK, Behari J. Influence of microwave exposure on fertility of male rats. Fertil Steril 2010; 95(4):1500-2.

[56] Moustafa YM, Moustafa RM, Belacy A, Abou-El-Ela SH, Ali FM. Effects of acute exposure to the radiofrequency fields of cellular phones on plasma lipid peroxide and antioxidase activities in human erythrocytes. J Pharm Biomed Anal 2001; 26: 605-8.

[57] Oral B, Guney M, Ozguner F, et al. Endometrial apoptosis induced by a $900-\mathrm{MHz}$ mobile phone: preventive effects of vitamins $\mathrm{E}$ and C. Adv Ther 2006; 23: 957-73.

[58] Ribeiro EP, Rhoden EL, Horn MM, Rhoden C, Lima LP, Toniolo L. Effects of subchronic exposure to radio frequency from a conventional cellular telephone on testicular function in adult rats. J Urol 2007; 177: 395-9.

[59] Dasdag S, Ketani MA, Akdag Z, et al. Whole-body microwave exposure emitted by cellular phones and testicular function of rats. Urol Res 1999; 27: 219-23.

[60] Dasdag S, Zulkuf Akdag M, Aksen F, et al. Whole body exposure of rats to microwaves emitted from a cell phone does not affect the testes. Bioelectromagnetics 2003; 24: 182-8.

[61] Alvarez JG, Touchstone JC, Blasco L, Storey BT. Spontaneous lipid peroxidation and production of hydrogen peroxide and superoxide in human spermatozoa. Superoxide dismutase as major enzyme protectant against oxygen toxicity. J Androl 1987; 8: 33848.

[62] Gavella M, Lipovac V. Antioxidative effect of melatonin on human spermatozoa. Arch Androl 2000; 44: 23-7.

[63] Burch JB, Reif JS, Noonan CW, et al. Melatonin metabolite excretion among cellular telephone users. Int J Radiat Biol 2002; 78: 1029-36.

[64] Zini A, Kamal K, Phang D, Willis J, Jarvi K. Biologic variability of sperm DNA denaturation in infertile men. Urology 2001; 58: 25861.

[65] Aitken RJ, Bennetts LE, Sawyer D, Wiklendt AM, King BV. Impact of radio frequency electromagnetic radiation on DNA integrity in the male germline. Int J Androl 2005; 28: 171-9.

[66] Falzone N, Huyser C, Fourie F, Toivo T, Leszczynski D, Franken D. In vitro effect of pulsed $900 \mathrm{MHz}$ GSM radiation on 
mitochondrial membrane potential and motility of human spermatozoa. Bioelectromagnetics 2008; 29: 268-76.

[67] Falzone N, Huyser C, Becker P, Leszczynski D, Franken DR. The effect of pulsed $900-\mathrm{MHz}$ GSM mobile phone radiation on the acrosome reaction, head morphometry and zona binding of human spermatozoa. Int J Androl 2011; 34: 20-6.

[68] Izard MA. Leydig cell function and radiation: a review of the literature. Radiother Oncol 1995; 34: 1-8.

[69] Zhou W, Wang XB, Yang JQ, Liu Y, Zhang GB. [Influence of electromagnetic irradiation on P450scc mRNA expression in rat testis tissues and protective effect of the shield]. Zhonghua Nan Ke Xue 2005; 11: 269-71.

[70] Wang S-M, Wang D-W, Peng R-Y, et al. [Effect of electromagnetic pulse irradiation on structure and function of Leydig cells in mice]. Zhonghua Nan Ke Xue 2003; 9: 327-30.

[71] Ozguner M, Koyu A, Cesur G, et al. Biological and morphological effects on the reproductive organ of rats after exposure to electromagnetic field. Saudi Med J 2005; 26: 405-10.

[72] Salama N, Kishimoto T, Kanayama H-O, Kagawa S. The mobile phone decreases fructose but not citrate in rabbit semen: a longitudinal study. Syst Biol Reprod Med 2009; 55: 181-7.

[73] Forgacs Z, Somosy Z, Kubinyi G, et al. Effect of whole-body $1800 \mathrm{MHz}$ GSM-like microwave exposure on testicular steroidogenesis and histology in mice. Reprod Toxicol 2006; 22: 111-7.

[74] Khillare BB, J. Effect of Amplitude-Modulated Radiofrequency Radiation on Reproduction Pattern in Rats. Electromagn Biol Med 1998; 17: 43-55

[75] Djeridane Y, Touitou Y, de Seze R. Influence of electromagnetic fields emitted by GSM-900 cellular telephones on the circadian patterns of gonadal, adrenal and pituitary hormones in men. Radiat Res 2008; 169: 337-43.

[76] Kesari KKBJ. Effects of microwave at $2.45 \mathrm{GHz}$ radiations on reproductive system of male rats. Toxicoll Environ Chem 2010; 92: 1135-47.

[77] Eberhardt JL, Persson BR, Brun AE, Salford LG, Malmgren LO. Blood-brain barrier permeability and nerve cell damage in rat brain 14 and 28 days after exposure to microwaves from GSM mobile phones. Electromagn Biol Med 2008; 27: 215-29.

[78] Wang XW, Ding GR, Shi CH, et al. Mechanisms involved in the blood-testis barrier increased permeability induced by EMP. Toxicology 2010;276: 58-63.

[79] Lievano S, Alarcon L, Chavez-Munguia B, Gonzalez-Mariscal L. Endothelia of term human placentae display diminished expression of tight junction proteins during preeclampsia. Cell Tissue Res 2006; 324: 433-48

[80] McCarthy KM, Francis SA, McCormack JM, et al. Inducible expression of claudin-1-myc but not occludin-VSV-G results in aberrant tight junction strand formation in MDCK cells. J Cell Sci 2000; 113 (Pt 19): 3387-98.

[81] Lui WY, Mruk D, Lee WM, Cheng CY. Sertoli cell tight junction dynamics: their regulation during spermatogenesis. Biol Reprod 2003; 68: 1087-97.

[82] Xiao-Wu Wang G-RD, Chang-Hong Shi, Tao Zhao, Jei Zhang, LiHua Zeng, Guo-Zhen Guo. Effect of Electromagnetic Pulse Exposure on Permeability of Blood-testicle Barrier in Mice1. Biomed Environ Sci 2008; 21:218-221.

[83] de Seze R, Fabbro-Peray P, Miro L. GSM radiocellular telephones do not disturb the secretion of antepituitary hormones in humans. Bioelectromagnetics 1998; 19: 271-8.

[84] Djeridane Y, Touitou Y, de Seze R. Influence of electromagnetic fields emitted by GSM-900 cellular telephones on the circadian patterns of gonadal, adrenal and pituitary hormones in men. Radiat Res 2008; 169: 337-43.

[85] Bortkiewicz A. A study on the biological effects of exposure mobile-phone frequency EMF. Med Pr 2001; 52: 101-6.

[86] Fang H-H, Zeng G-Y, Nie Q, et al. Effects on structure and secretion of pituitary gland in rats after electromagnetic pulse exposure. Zhonghua Yi Xue Za Zhi 2010; 90: 3231-4

[87] Yan J-G, Agresti M, Bruce T, Yan YH, Granlund A, Matloub HS Effects of cellular phone emissions on sperm motility in rats. Fertil Steril 2007; 88: 957-64.

[88] Eltiti S, Wallace D, Ridgewell A, et al. Does short-term exposure to mobile phone base station signals increase symptoms in individuals who report sensitivity to electromagnetic fields? A double-blind randomized provocation study. Environ Health Perspect 2007; 115: 1603-8.

[89] Goldsworthy A. The Cell Phone and the Cell. 1st Hellenic Congress - The Effects of Electromagnetic Radiation 2008.

[90] Mouradi RD, Erdemir NA, Ashok A. The Use of FDTD in establishing In-vitro experimentation conditions representative of lifelike cell phone radiation on the spermatozoa. 2010 [Unpublished data].

[91] Makker K, Varghese A, Desai NR, Mouradi R, Agarwal A. Cell phones: modern man's nemesis? Reprod Biomed Online 2009; 18 $148-57$. 